\title{
New opportunities for AI EDAM: Ecological systems and biological designs
}

\author{
ASHOK K. GOEL \\ School of Interactive Computing, Georgia Institute of Technology, Atlanta, Georgia, USA
}

I begin by thanking the outgoing editor David Brown for two major responsibilities. In one, Dave acted as a "seer," identifying important research goals, themes, and trends and publishing special issues on them. This included core themes of fundamental value such as functional reasoning and multimodal representations, as well as emerging trends of significant promise such as creative cognition and biologically inspired design. These special issues often have had a long-term impact that is not easily measured by "impact factors." In the second role, Dave acted as a "gatekeeper," carefully screening papers, for example, papers on routine applications of genetic algorithms, neural networks, and other function optimization techniques that can overwhelm an artificial intelligence (AI) journal but provide only modest insights into engineering design, analysis, and manufacturing (EDAM).

The last 10 years have witnessed important changes both in AI and EDAM, the two halves of AI EDAM. In the 1990s, the amount of research on knowledge-based AI and cognitively oriented AI gradually declined. However, over the last decade, this kind of AI has remerged as interactive AI, or what I will call human-centered AI: AI that is situated among humans and interactively serves them. Human-centered AI is knowledge based and cognitively oriented. It is also closely integrated with interactive techniques from computational media, human-computer interaction, machine learning, and so on. Further, until the early 1990s, AI research was conducted mostly in computer science departments; but over the last couple of decades, AI research has permeated many good EDAM schools as well. I am pleased that this journal has assimilated both of these changes quite well. For exam- ple, an increasing number of special issues now focus on various aspects of human-centered AI in EDAM.

From my perspective, the ongoing changes in EDAM are even more exciting and bigger scale. First, almost all EDAM over the last century has been based on the physical sciences. However, this is changing. As Charles Vest, President, US National Academy of Engineering, and President Emeritus of MIT, recently said (http://www.nytimes.com/ interactive/2010/11/09/science/): "We're going to see in surprisingly short order that biological inspiration and biological processes will become central to engineering real systems. It's going to lead to a new era in engineering." Although the physical sciences certainly will continue to be very important, I expect that biological sciences will soon begin to play a critical role in engineering, leading to new methods and practices of biologically inspired engineering design and manufacturing. Second, in the big picture, much of EDAM in the past has been driven by considerations of economy and culture. Although economy and culture of course will continue to be critical determinants, limitations of natural resources and ecological sustainability will become increasingly important in EDAM. I posit that because of these two developments, AI research on EDAM in the next decade will address problems quite different from those, say, in the 1990s: the new set of problems will engage biology, ecology, and the environment at a depth and scale never before seen in this journal.

These rapid and important changes in AI, and especially in EDAM, make this is a time of significant opportunities and challenges for $A I E D A M$. In working with the incoming editor Yan Jin, I look forward to helping AI EDAM exploit these opportunities. 